\title{
Heterochromatin assembly and transcriptome repression by Set 1 in coordination with a class II histone deacetylase
}

\author{
David R Lorenz, Lauren F Meyer, Patrick J R Grady, Michelle M Meyer, \\ Hugh P Cam*
}

Department of Biology, Boston College, Chestnut Hill, United States

\begin{abstract}
Histone modifiers play essential roles in controlling transcription and organizing eukaryotic genomes into functional domains. Here, we show that Set1, the catalytic subunit of the highly conserved Set1C/COMPASS complex responsible for histone H3K4 methylation (H3K4me), behaves as a repressor of the transcriptome largely independent of Set1C and H3K4me in the fission yeast Schizosaccharomyces pombe. Intriguingly, while Set1 is enriched at highly expressed and repressed loci, Set1 binding levels do not generally correlate with the levels of transcription. We show that Set1 is recruited by the ATF/CREB homolog Atf1 to heterochromatic loci and promoters of stress-response genes. Moreover, we demonstrate that Set1 coordinates with the class II histone deacetylase Clr3 in heterochromatin assembly at prominent chromosomal landmarks and repression of the transcriptome that includes Tf2 retrotransposons, noncoding RNAs, and regulators of development and stress-responses. Our study delineates a molecular framework for elucidating the functional links between transcriptome control and chromatin organization.
\end{abstract}

DOI: 10.7554/eLife.04506.001

*For correspondence: hugh. cam@bc.edu

Competing interests: The authors declare that no competing interests exist.

Funding: See page 13

Received: 26 August 2014 Accepted: 12 December 2014 Published: 15 December 2014

Reviewing editor: Ali Shilatifard, Northwestern University Feinberg School of Medicine, United States

(c) Copyright Lorenz et al. This article is distributed under the terms of the Creative Commons Attribution License, which permits unrestricted use and redistribution provided that the original author and source are credited.

\section{Introduction}

The packaging of eukaryotic DNA with histones into chromatin provides ample opportunities for chromatin-modifying factors to exert extensive control over many aspects of genome-based processes (Kouzarides, 2007). In particular, enzymes catalyzing the covalent posttranslational modifications of histones are increasingly seen as critical regulators of transcription and the assembly of chromatin into various functional domains (Henikoff and Shilatifard, 2011; Badeaux and Shi, 2013). Two of the better understood posttranslational modifications of histones are acetylation and methylation. Whereas acetylation of histones by histone acetyltransferases (HATs) is generally associated with gene activation (Rando and Chang, 2009), deacetylation of histones by histone deacetylases (HDACs) tends to correlate with gene repression (Yang and Seto, 2008). Coordinated activities among HATs result in region-wide hyperacetylated chromatin states, leading to the formation of euchromatin domains supporting active transcription, and conversely, hypoacetylated chromatin states catalyzed by HDACs give rise to heterochromatin domains refractory to transcription (Grunstein, 1998; Grewal and Jia, 2007). In contrast, histone methylation is associated with either transcriptional activation or repression, and hence, with euchromatin or heterochromatin (Huisinga et al., 2006; Henikoff and Shilatifard, 2011). Two well-characterized methylation marks occurring on two closely spaced residues near the amino-terminal tail of histone H3 exemplify this pattern (Grewal and Jia, 2007). Methylation at lysine 4 of histone $\mathrm{H} 3$ (H3K4me) and at lysine 9 ( $\mathrm{H} 3 \mathrm{~K} 9 \mathrm{me})$ distinguishes euchromatin and heterochromatin, respectively (Litt et al., 2001; Noma et al., 2001). However, studies from the fission yeast Schizosaccharomyces pombe and other systems show that the euchromatic and heterochromatic landscapes are somewhat fluid, with islands of H3K9me transiently assembled within 
eLife digest Genes can be turned on or off at different times in an organism's life. In humans, yeast and other eukaryotes, this is mainly controlled by the way DNA is packaged with proteinsknown as histones-in a structure called chromatin. Genes that are switched on, or only temporarily switched off, are associated with areas of the genome where the chromatin is loosely packed. In contrast, genes that remain switched off for long periods of time are found in regions-known as heterochromatin-where the chromatin is tightly packed.

There are many enzymes that can modify histones to change the structure of chromatin. One enzyme - called Set1—adds a methyl tag to chromatin, which is known to be associated with genes being switched on. However, Lorenz et al. found that Set1 also has other roles in modifying chromatin in the yeast Schizosaccharomyces pombe.

The experiments found that Set1 helps to keep genes switched off and that this role is largely independent of its ability to add the methyl tag to chromatin. Set1 is recruited to many sites across the genome by another protein called Atf1, which is involved in the cell's response to environmental stresses. Lorenz et al. believe that this helps to put these genes in a 'poised' off state so that they are ready to be switched on rapidly if needed.

Set1 also works with another protein that removes acetyl tags-which encourage chromatin to be less tightly packed-from histones. Together, both proteins contribute to the assembly of heterochromatin and keep genes involved in development and stress responses switched off when they are not required.

Collectively, these experiments reveal unexpected and important insights into how Set1-which plays critical roles in many aspects of human health including aging and cancer-works in cells. DOI: 10.7554/eLife.04506.002

euchromatin at certain meiotic genes and the $3^{\prime}$ ends of convergent genes (Cam et alo, 2005; Huisinga et al., 2006; Gullerova and Proudfoot, 2008; Zofall et al., 2012; Tashiro et al., 2013). Conversely, the RNA interference (RNAi) and exosome machineries, certain HATs and an active RNA polymerase II (Pol II) have been documented to contribute directly to the assembly of heterochromatin (Volpe et al., 2002; Djupedal et alı, 2005; Kato et al., 2005; Buhler et al., 2007; Xhemalce and Kouzarides, 2010; Reyes-Turcu et al., 2011; Yamanaka et al., 2013).

These observations point to the potential roles for other chromatin-modifying factors normally associated with euchromatin in heterochromatin assembly. In particular, the Saccharomyces cerevisiae homolog of Set1 (KMT2) responsible for H3K4 methylation (H3K4me) has been implicated in transcriptional silencing at a number of genetic elements (Nislow et al., 1997; Krogan et al., 2002; Berretta et al., 2008; Camblong et al., 2009; Kim and Buratowski, 2009; van Dijk et al., 2011). Set1 forms the catalytic engine of a highly conserved chromatin-modifying complex termed Set1C or COMPASS (Shilatifard, 2012). Set1C subunits have been shown to be recruited to active Pol Il genes and provide the H3K4me signature for the gene-rich euchromatin (Krogan et al., 2003; Ng et al., 2003). H3K4me can exist in a mono- (H3Kme1), di- (H3K4me2), or tri- (H3K4me3) methylated form (Kusch, 2012). The three forms of H3K4me have different distributions, with $\mathrm{H} 3 \mathrm{~K} 4 \mathrm{me} 3$ and $\mathrm{H} 3 \mathrm{~K} 4 \mathrm{me} 2$ enriched at gene promoters and gene bodies, respectively (Cam et al., 2005; Pokholok et al., 2005). H3K4me1 is enriched at the $3^{\prime}$ end of Pol II genes in budding yeast and at enhancers in mammals (Pokholok et al., 2005; Heintzman et al., 2007). Gene expression profiling analyses ascribe the repressor function of Set1C to H3K4me2 and/or H3K4me3 (Margaritis et al., 2012; Weiner et al., 2012).

We have recently discovered a role for the $S$. pombe Set1 in the transcriptional repression and genome organization of long terminal repeat $T f 2$ retrotransposons and heterochromatic repeats that are dependent and independent of the Set1C complex and H3K4 methylation (Lorenz et al., 2012; Mikheyeva et al., 2014). In this study, we investigate the regulatory control of the fission yeast transcriptome by Set1 and its associated Set1C subunits. By systematically analyzing the transcriptomes of H3K4me mutants and mutant strains deficient in each of the Set1C subunits, we find that even though loss of H3K4me generally results in derepression, Set1 exerts its repressive function on most of its targets largely independently of the other Set1C subunits and H3K4me. Intriguingly, genome-binding profiles showed that Set1 localization is not linearly correlated with the levels of transcription at its target loci. In addition to localization at active Pol II genes, Set1 localizes to repetitive elements and 
repressed loci associated with development and stress-response pathways. Furthermore, we demonstrate that the conserved stress-response ATF/CREB Atf1 transcription factor mediates the recruitment of Set 1 and modulates the levels of $\mathrm{H} 3 \mathrm{~K} 4 \mathrm{me} 3$ at the centromere central cores and ribosomal DNA array. We show that Set1 coordinates with the class II HDAC Clr3 to mediate the assembly of H3K9me-associated heterochromatin and genome-wide repression of diverse transcripts, including Tf2 retrotransposons, noncoding RNAs, and developmental and stress-response genes. Our study illuminates a surprising cooperation between two histone-modifying enzymes with seemingly opposing activities in imposing genome-wide repression over the transcriptome and organizing the genome into euchromatin and heterochromatin.

\section{Results}

\section{Set1 behaves as a general repressor largely independent of its H3K4me function and other Set1C subunits}

Set1 is the catalytic engine of the Set1C complex that includes seven other subunits (Roguev et al., 2003). Except for Shg1, Set1 and six S. pombe subunits (Swd1, Swd2, Swd3, Spp1, Ash2, Sdc1) have orthologs in S. cerevisiae and humans (Roguev et al., 2003; Shevchenko et al., 2008; Shilatifard, 2012). Loss of individual Set1C complex subunits affects differentially the levels and states of H3K4me in S. pombe (Roguev et al., 2003; Mikheyeva et al., 2014). We performed expression profiling analyses in mutant strains deficient in $\mathrm{H} 3 \mathrm{~K} 4 \mathrm{me}$ or lacking individual subunits of the Set1C complex. Whereas loss of set 1 resulted in significant derepression of nearly 1000 of $\sim 42,000$ tiling microarray probes (average $\log _{2}$ fold-change vs wild-type $>1.5, \mathrm{p}<0.05$ ), H3K4me null mutants H3K4R (histone H3 lysine

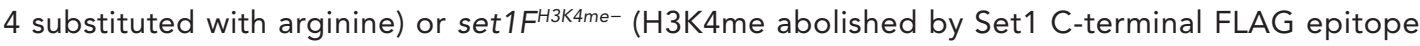
insertion) (Lorenz et al., 2012; Mikheyeva et al., 2014) affected 100 probes (Figure 1A). Profiling analysis of other Set1C subunits showed a wide range of effects on transcriptional repression, with fewer than 100 probes significantly changed versus wild-type in ash $2 \Delta$ to $\sim 300$ in spp $1 \Delta$. Similar to the other H3K4me mutants, most probes affected in Set1C subunit mutants corresponded to upregulated transcripts, consistent with previous observations in budding yeast showing that loss of $\mathrm{H} 3 \mathrm{~K} 4 \mathrm{me}$ tends to result in derepression (Margaritis et al., 2012; Weiner et al., 2012). Importantly, our results show that the major repressive function of Set1 in S. pombe occurs largely distinct from H3K4me and the Set1C complex. Variations among Set1C/H3K4me mutants in the proportion of affected probes corresponding to sense, antisense, and intergenic transcripts were also observed (Figure 1B), with equal proportions of differentially expressed probes among the three classes of transcripts seen in set $1 \Delta$, $H 3 K 4 R$, and set $1 \mathrm{~F}^{H 3 K 4 m e-}$ mutants. Loss of ash2 primarily resulted in increased sense transcription, and loss of shg1, spp1, or swd3 predominantly affected intergenic transcripts.

\section{Set1C/H3K4me mutants display unique gene expression profiles}

Because Set1C/H3K4me mutants displayed varying degrees of transcriptional effects, we performed two-dimensional hierarchical clustering of all differentially expressed probes to gain further insights into their functional relationships. Despite their functions being linked to H3K4me, transcriptional profiles clustered broadly into four distinct groups (Figure 1C, upper panel). The loss of ash2 and sdc1, which affected a higher proportion of sense strand probes than in other mutants (Figure 1B), shared a subset of upregulated transcripts with significant gene ontology (GO) enrichment for terms common to stress response, including 'response to stress' $\left(p \approx 10^{-3}\right.$, ash $2 \Delta ; p \approx 10^{-18}$, sdc $\left.1 \Delta\right)$, 'oxidoreductase activity' $\left(p \approx 10^{-3}\right.$, ash $2 \Delta ; p \approx 10^{-11}$, sdc $\left.1 \Delta\right)$, and 'generation of precursor metabolites and energy' $\left(p \approx 10^{-5}\right.$, ash2 $\Delta, p \approx 10^{-3}, s d c 1 \Delta$ ) (Figure 1-source data $1 A$ ). The profiles of shg1 and spp1 mutants formed the second group of predominantly upregulated probes corresponding to diverse intergenic regions and antisense transcripts sharing comparatively weak GO enrichment. The group consisting of swd1 $\Delta$, swd2 2 , swd $3 \Delta$, set1 $\mathrm{F}^{\mathrm{H} K 4 \mathrm{Kme}}$, and $H 3 K 4 R$ mutants included smaller subsets of differentially expressed probes (Figure 1C, upper panel), with modestly significant GO enrichment for upregulated transcripts related to stress response and carbohydrate metabolism (Figure 1-source data 1A). The profile of set1 $\Delta$ forms its own distinct group, containing a large set of upregulated transcripts including Tf2 retrotransposons, pericentromeric repeats, and long noncoding RNAs (IncRNAs) that were little affected in the other Set1C and H3K4me mutants (Figure 1C, lower panel; Figure 1-source data 1B). These results suggest that loss of individual Set1C subunits produces different effects on the transcriptome that could not be fully accounted for by their known contributory roles to H3K4 methylation. 
A

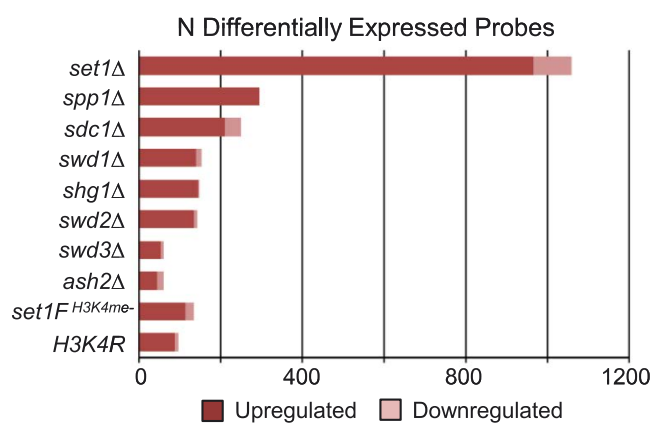

B

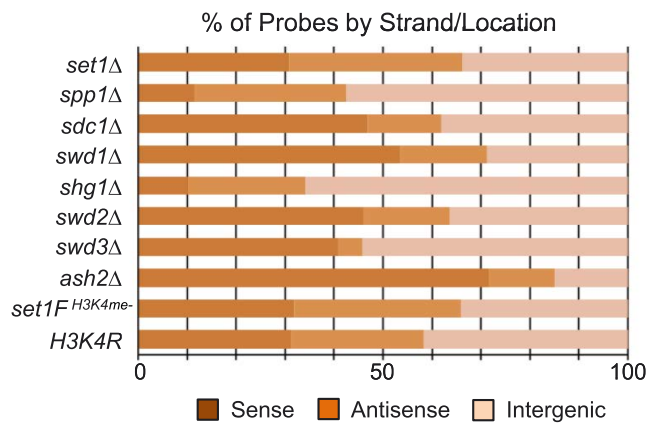

C

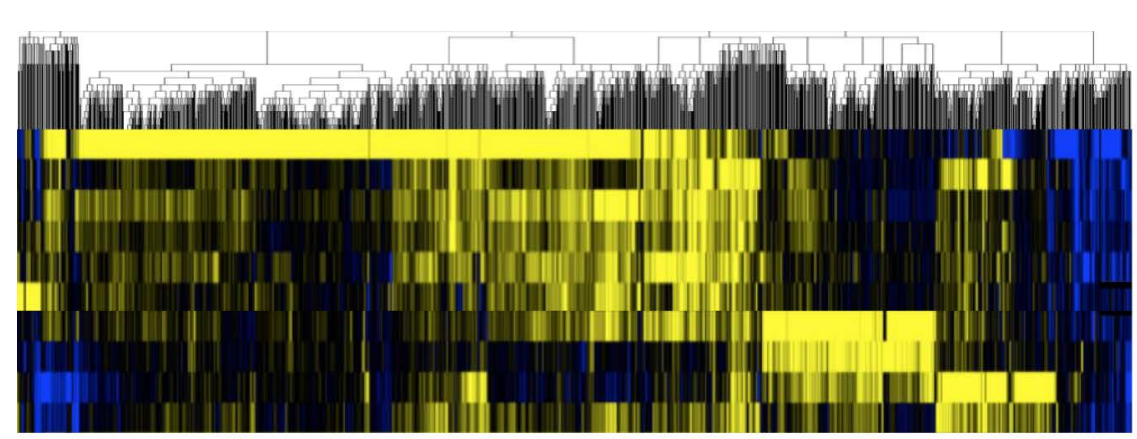

$\triangle$ mRNA vs wt $\left(\log _{2}\right)$

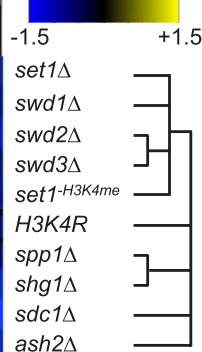

Probe Location

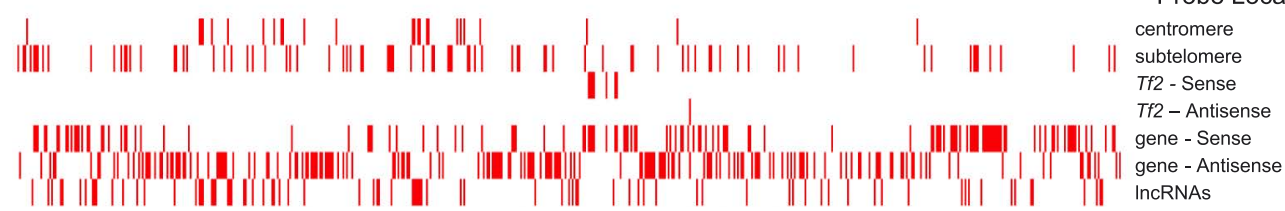

Figure 1. Set1/COMPASS subunits act primarily as transcriptional repressors. (A) Counts and (B) percentage of probes by matching feature strand/position of differentially expressed probes from custom 44,000-probe tiling microarrays. Significantly changed probes were defined as absolute $\log _{2}$ fold-changes $\geq 1.5$, false discovery rate (FDR)-adjusted $p$ values $<0.05$ from duplicate arrays. (C) Hierarchical clustering of differentially expressed probes (absolute $\log _{2}$ fold-change vs wild-type $\geq 1.5, \mathrm{p}<0.05$ ) in Set1C/H3K4me mutant strains. Probes showing significant expression changes in the indicated mutant versus wild-type strains were clustered using the HOPACH algorithm. The bottom panel shows the positions of probes matching repetitive centromeric, subtelomeric (100,000 bp end sequences of all chromosomes), Tf2 retrotransposons, the sense or antisense strands of annotated protein coding genes, or intergenic long noncoding RNAs (IncRNAs).

DOI: 10.7554/eLife.04506.003

The following source data is available for figure 1:

Source data 1. Gene ontology (GO) enrichment in Set1C/COMPASS mutant expression profiling microarrays. DOI: 10.7554/eLife.04506.004

Source data 2. Comparative analysis of common enriched GO terms in Set1C/COMPASS mutant expression profiling microarrays.

DOI: 10.7554/eLife.04506.027

\section{Set1 localizes to lowly expressed and repressed genes}

While H3K4me is known to be enriched at transcriptionally active loci (Cam et alo, 2005; Pokholok et al., 2005), we consistently observed transcriptional derepression in the set1 $\Delta$ mutant at non-active, stress-response genes or heterochromatic repeats. We therefore performed genome-wide mapping of Set1 to gain insights into its repressor function. Consistent with its documented recruitment to active Pol Il genes ( $\mathrm{Ng}$ et al., 2003), Set1 is enriched at sites that correspond to highly active Pol II promoters, including those of the housekeeping gene act1 and the ribosomal protein rps102 (Figure 2A). 


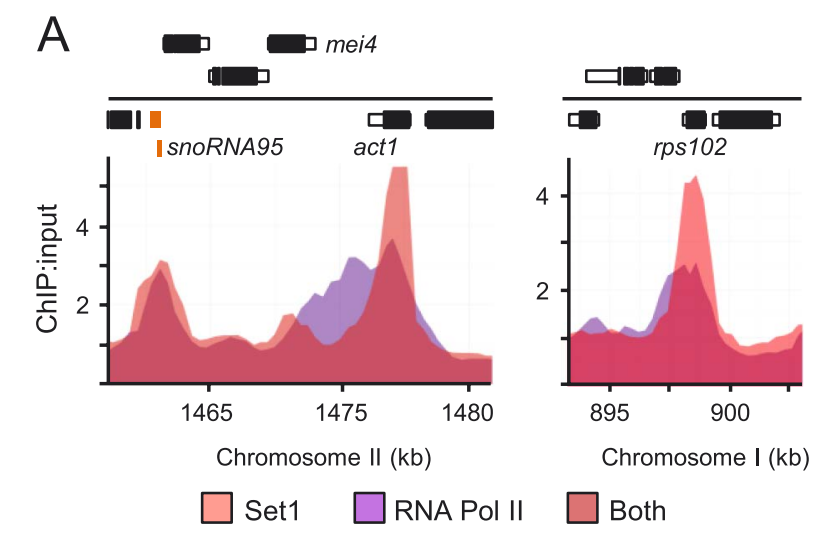

C

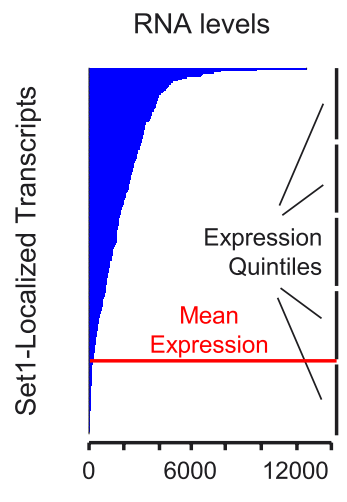

(FPKM)
Pol II ChIP

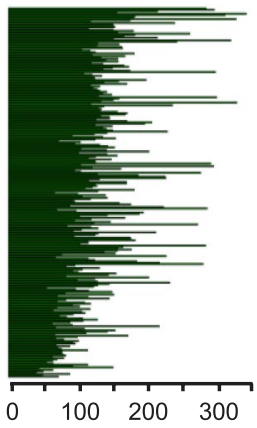

(FPM)

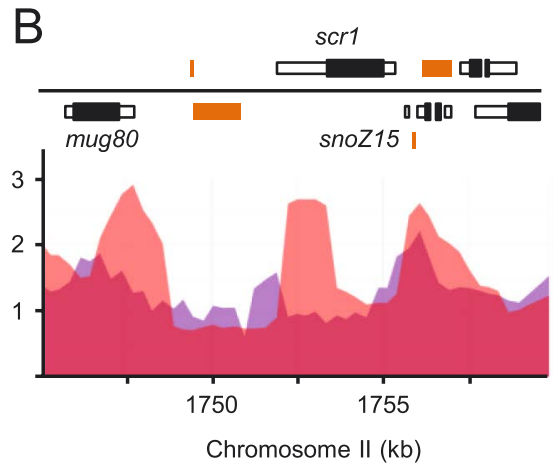

Set1
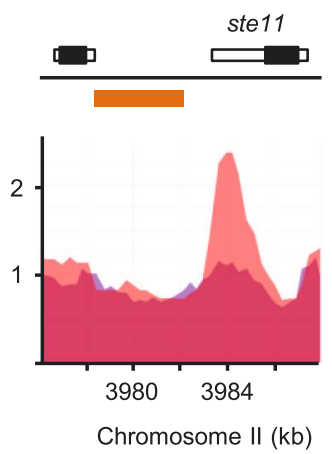

Both

D

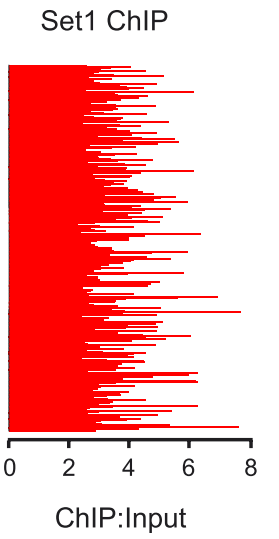

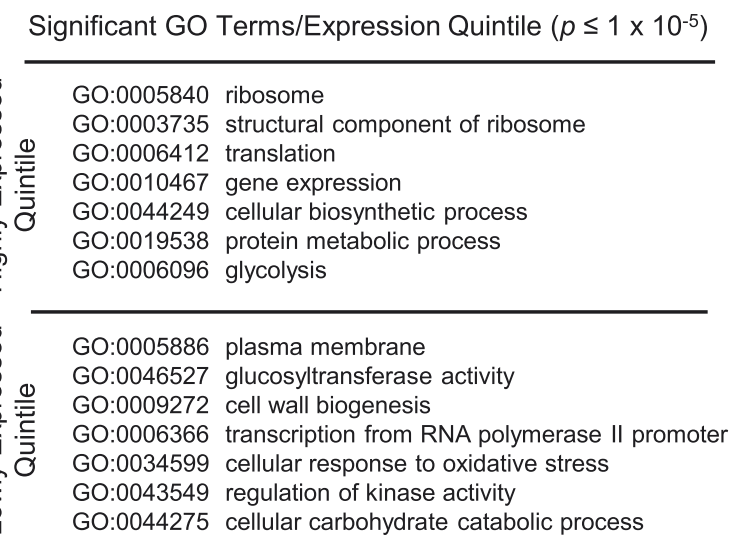

Figure 2. Set1 localizes to lowly expressed and repressed loci. (A and B) Enrichment of Set1 and RNA polymerase II (Pol II) determined by chromatin immunoprecipitation (ChIP)-chip displaying significant Set1 enrichment at highly transcribed genes (A) and repressed genes (B). Positions of genomic features on forward (top) and reverse strands (bottom), top panel. Black bars denote protein coding gene open reading frames (ORFs); white, associated untranslated regions (UTRs); orange, noncoding RNAs. Pol II ChIP-chip data was derived from Chen et al. (2008). (C) Set1 enrichment relative to transcript abundance and Pol II occupancy. Comparisons of RNA-seq expression levels (blue), Pol II ChIP-seq enrichment (green) and Set1 ChIP-chip enrichment (red) at loci showing significant Set1 enrichment ( $N=290$ transcripts with nonoverlapping annotated features). Processed RNA-Seq FPKM data were obtained from Rhind et al. (2011) and Pol II ChIP-seq data from Zaratiegui et al. (2010). The horizontal red line denotes mean expression for all Schizosaccharomyces pombe transcripts (Rhind et al., 2011). (D) Gene ontology (GO) analysis of Set1-bound transcripts by expression level quintile. Representative GO terms were significantly enriched ( $p \leq 1 \times 10^{-5}$, hypergeometric test) and found exclusively in quintiles of highly expressed (top panel) versus lowly expressed genes (bottom panel). See Figure 2-source data 1 for a complete list of all significantly enriched GO terms/quintile. DOI: 10.7554/eLife.04506.005

The following source data and figure supplements are available for figure 2:

Source data 1. Gene ontology (GO) enrichment of Set1-localized transcripts (ChIP-chip) by target expression level. DOI: 10.7554/eLife.04506.006

Figure supplement 1. Set1 localization at active and repressed loci. DOI: 10.7554/eLife.04506.007

Figure supplement 2. Distribution of Set1-localized versus all Schizosaccharomyces pombe transcripts by absolute expression level. DOI: 10.7554/eLife.04506.008

Surprisingly, despite little enrichment of Pol II at certain lowly expressed genes (e.g., scr1) and repressed developmental genes (e.g., ste11), noticeable Set1 binding was detected at the promoters of these genes (Figure 2B; Figure 2-figure supplement 1). Set1 localization at active and repressed targets was not hampered by the loss of H3K4me or its catalytic activity. Indeed, the inability of the set $1 \mathrm{~F}^{\mathrm{H} K 4 m e-}$ to methylate $\mathrm{H} 3 \mathrm{~K} 4$ appears to enhance its association with chromatin. To discern the relationship between Set1 binding and the transcriptional status of its targets, we ranked 290 protein-coding genes with significant Set1 binding (chromatin immunoprecipitation 
(ChIP) fold enrichment $\geq 2$ at three or more adjacent probes) according to their expression levels (Figure 2C, left panel). While transcript abundance generally correlated with Pol Il occupancy levels (Figure $2 \mathrm{C}$, middle panel) and $80 \%$ of promoter regions enriched for Set 1 corresponded to actively transcribed genes (Figure 2-figure supplement 2), transcript abundance or Pol Il occupancy levels did not linearly correlate with the levels of Set1 binding (Figure 2C, right panel). Functional differences between high-abundance and low-abundance Set1-bound genes were assessed by GO analysis of genes rank-ordered by expression levels into quintiles (Figure 2D). Whereas highly expressed genes occupied by Set1 were enriched with expected GO terms associated with rapid exponential growth (ribosome, translation, glycolysis), Set1-bound genes with low abundance transcripts (excluding heterochromatic noncoding RNAs due to limited GO annotation) were enriched for terms related to stress response, cell wall and membrane-bound protein biogenesis, and Pol II transcription factor function (Figure 2-source data 1). Thus, our results suggest that Set1 localization at chromatin is not solely dependent on active Pol II, and that Set1 localization at lowly expressed or repressed loci might be functionally distinct from its canonical role at active Pol Il genes.

\section{Atf1 mediates recruitment of Set1 at the centromere central cores, rDNA array, and developmental and stress-response genes}

A number of low-abundance transcripts shown to be enriched for Set1 in genome-wide binding profiling (e.g., ste11) have previously been shown to be targets of the highly conserved ATF/CREB transcription factor Atf1. In addition to localizing to its targets before their activation (Eshaghi et alo, 2010), which is important for subsequent proper response to environmental stresses (Chen et alo, 2003), Atf1 contributes to heterochromatic silencing at the silent mating-type locus (Jia et al., 2004). We performed genome-wide binding profiling of Atf1 and compared it with that of Set1 to gain insights into the mechanism of Set1 recruitment to chromatin. We observed colocalization of Atf1 and Set1 at centromeric tRNA clusters flanking the euchromatin/heterochromatin boundaries of centromere II and the inner imr repeats of the central core (Figure 3A, upper panel). Similar colocalization patterns were detected at centromeres I and III (Figure 3-figure supplement 1, upper panels). We also detected colocalization of Atf1 and Set1 at the intergenic region of the rDNA and the promoter of the developmental regulator ste11 (Figure 3B,C, upper panel; Figure 3-figure supplement 2). We assessed the loss of atf1 on Set1 activity by mapping distributions of $\mathrm{H} 3 \mathrm{~K} 4 \mathrm{me} 3$ at these loci in wildtype and atf1 $\Delta$ cells. In wild-type cells, $\mathrm{H} 3 \mathrm{~K} 4 \mathrm{me} 3$ signals could be detected throughout the centromere central cores and the rDNA array but were little enriched the ste11 promoter (Figure $3 A, B, C$; Figure 3-figure supplement 1, bottom panels). Loss of atf1 resulted in a sizeable reduction of H3K4me3 levels throughout the central cores and rDNA array. Moreover, genome-wide analysis identified many loci displaying reduced $\mathrm{H} 3 \mathrm{~K} 4 \mathrm{me} 3$ in atf1 $\Delta$ compared with wild-type (Figure 3-source data 1). The repressed status of the ste11 gene was not noticeably affected by atf1 (Figure 3-figure supplement 4) and hence has little effect on the status of H3K4me3. However, we noticed that several repressed genes whose promoters are occupied by Atf1 exhibited increased H3K4me3 levels in atf1 $\Delta$ cells (Figure 3-figure supplement 3), probably owing to the loss of Atf1-mediated repression.

To determine whether reduced H3K4me3 levels at the centromere central cores and the rDNA array partly reflect the failure of Atf1 to recruit Set1, we assessed Set1 localization at these loci by ChIP. We found that Set1 enrichment at these loci, including the ste11 gene, was reduced in atf1 $\Delta$ cells (Figure 3D). At ste11, Atf1 and Set1 appear to act primarily in parallel pathways to keep ste11 expression repressed, as appreciable upregulation of ste 11 expression was seen only in mutants deficient for both atf1 and set1 (Figure 3-figure supplement 4). Comparing Atf1 and Set1 localization at the genome scale revealed 217 and 261 distinct bound loci for Atf1 or Set1, respectively, with more than one-third cooccupied by both proteins ( $p<0.001$, Fisher's exact test) (Figure 3E). Collectively, our results suggest that Set1 recruitment to certain repressed loci is mediated in part by Atf1, which in turn is important for proper maintenance of $\mathrm{H} 3 \mathrm{~K} 4 \mathrm{me}$ levels and, depending on genomic context, transcriptional repression.

\section{Set1 cooperates with the class II HDAC Clr3 in heterochromatic silencing and the assembly of heterochromatin}

To better understand the repressive function of Set1, we sought to identify factors that cooperate with Set1 in heterochromatic silencing. The class II HDAC Clr3 has been shown to contribute to transcriptional silencing of heterochromatin (Grewal et al., 1998; Yamada et al., 2005) Tf2 retrotransposons (Hansen et al., 2005; Cam et al., 2008), and stress-response genes (Lorenz et al., 2012). These classes of 


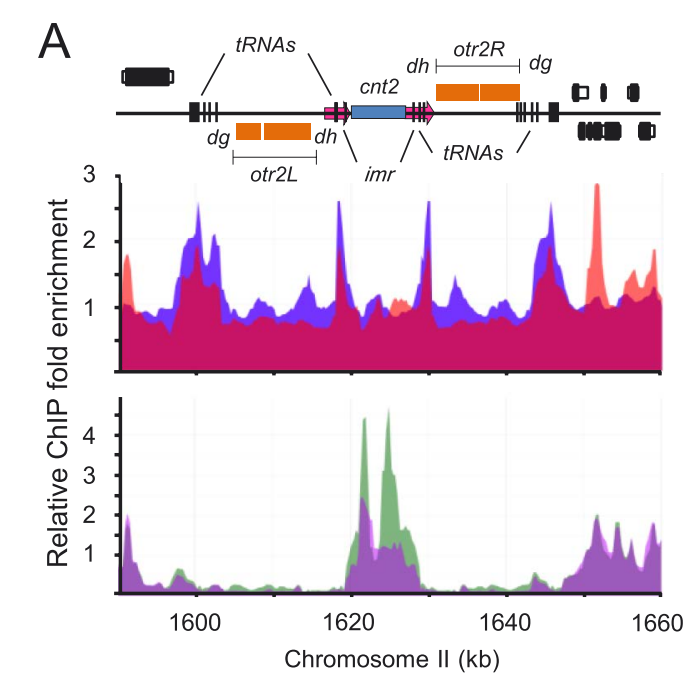

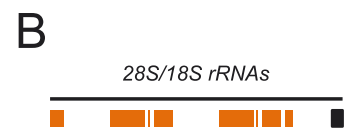
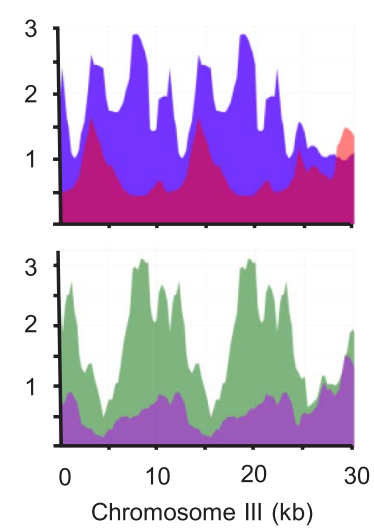
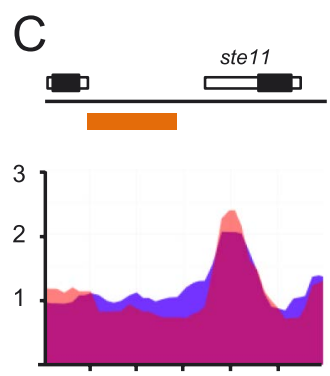

Set1

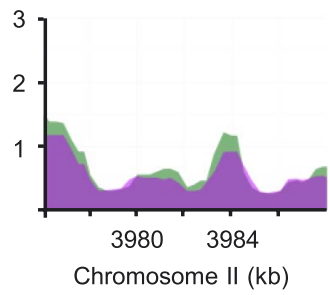

Chromosome II $(\mathrm{kb})$
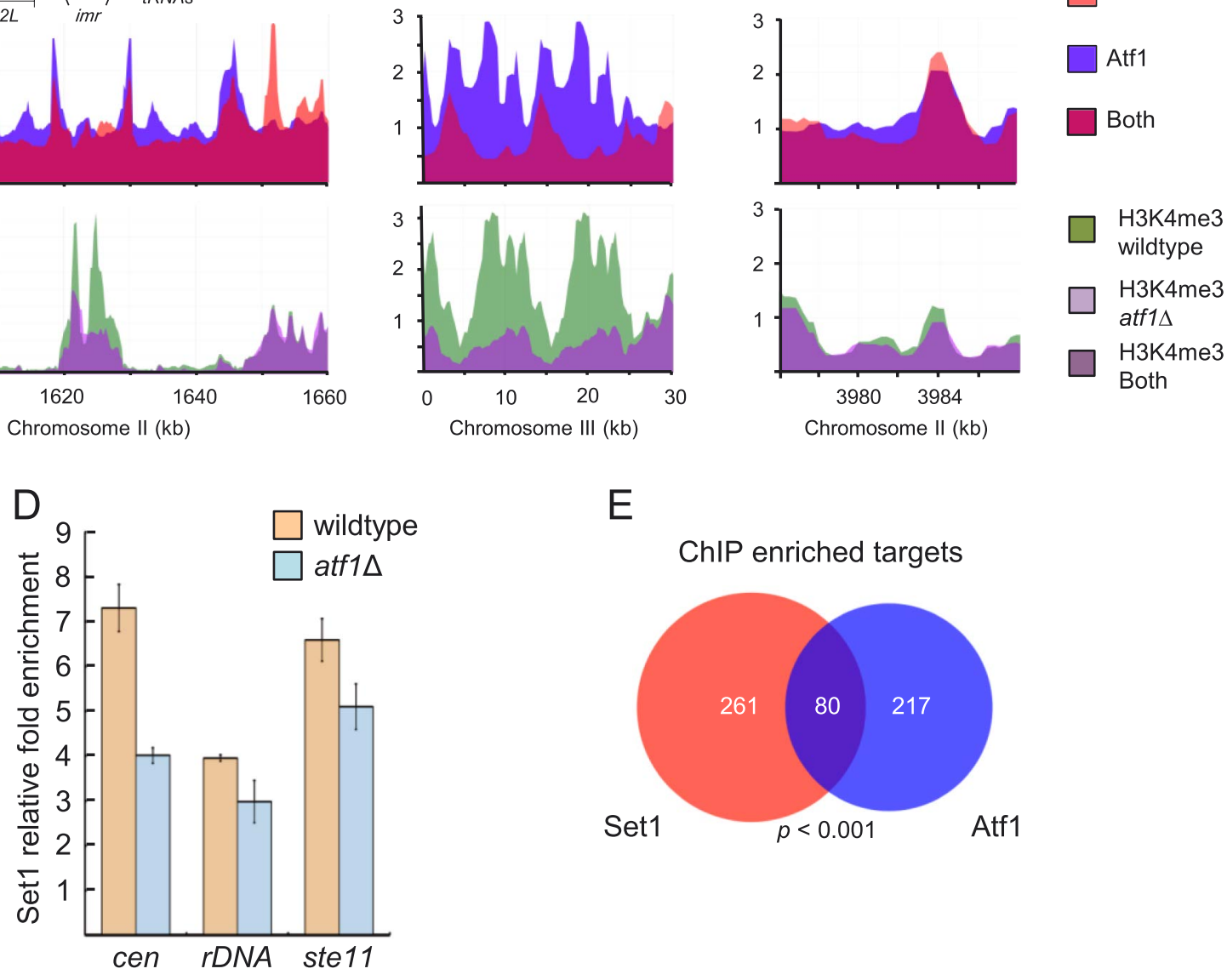

$\mathrm{E}$

\section{ChIP enriched targets}

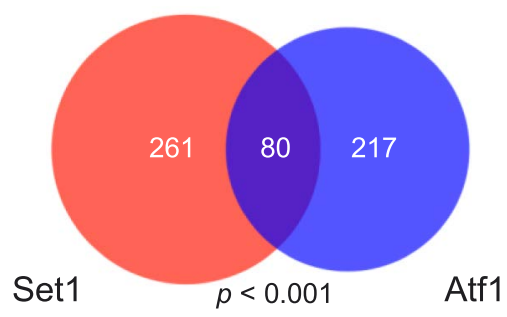

Figure 3. Atf1 mediates recruitment of Set1 to centromeres, rDNA, and ste11 and contributes to H3K4 methylation. (A) Colocalization of Atf1 and Set1 (upper panels) at centromere II, (B) rDNA array, and (C) the promoter of the developmental regulator ste11. Enrichment of H3K4me3 (A-C, lower panels) and Set1 (D) at the aforementioned loci in wild-type and atf1 $\Delta$ cells. Enrichment of Set1, Atf1 and H3K4me3 at indicated loci (A-C) was done by chromatin immunoprecipitation (ChIP)-chip. (E) Set1 and Atf1 regulate a common set of targets. Venn diagram of Atf1 and Set1 ChIP-chip peaks. Peaks were deemed overlapping if found within $1 \mathrm{~kb}$ of each other. The $\mathrm{p}$ value was determined by a hypergeometric test with population size $N=3667$ Schizosaccharomyces pombe intergenic regions.

DOI: 10.7554/eLife.04506.009

The following source data and figure supplements are available for figure 3:

Source data 1. Differential enrichment of H3K4me3 levels in atf1 $\Delta$ vs. wild-type cells.

DOI: 10.7554/eLife.04506.010

Figure supplement 1. Colocalization of Set1 and Atf1 at centromeres I and III.

DOI: 10.7554/eLife.04506.011

Figure supplement 2. Enrichment of Atf1 at repressed loci.

DOI: 10.7554/eLife.04506.012

Figure supplement 3. Atf1 acts as a transcriptional repressor.

DOI: 10.7554/eLife.04506.013

Figure supplement 4. Derepression of ste 11 in mutants deficient in both atf1 and set1.

DOI: 10.7554/eLife.04506.014

genetic elements are also regulated by Set1, suggesting a possible functional link between $\mathrm{Clr} 3$ and Set1. To explore this idea, we constructed a mutant strain deficient for both set1 and $\operatorname{clr} 3$ ( $\operatorname{set} 1 \Delta \operatorname{clr} 3 \Delta$ ). We observed that in contrast to wild-type or single set1 $\Delta$ or clr3 $\Delta$ mutant strains, a double mutant set1 $\Delta$ clr3 $\Delta$ strain exhibited a significant synthetic slow-growth phenotype and sensitivity to the tubulin 
A $\mathrm{N} / \mathrm{S}$

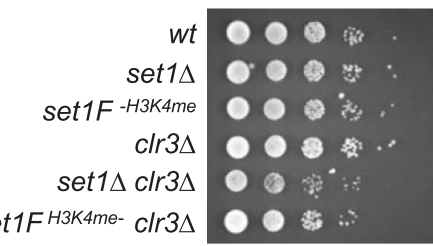

B

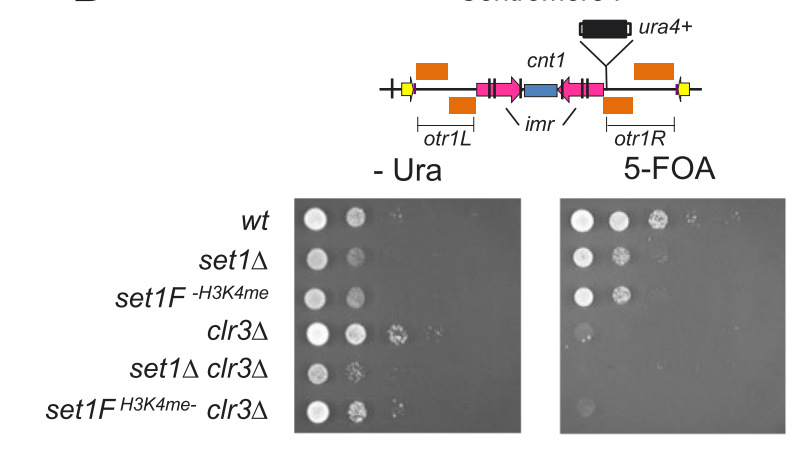

C

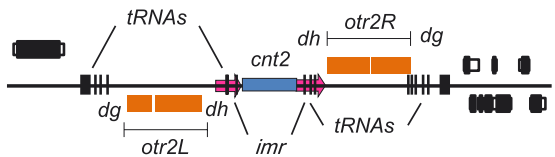

Forward Strand

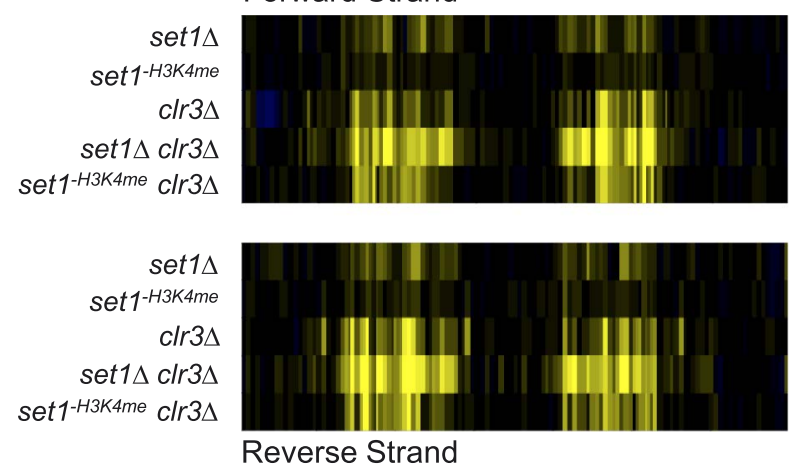

Reverse Strand

TBZ

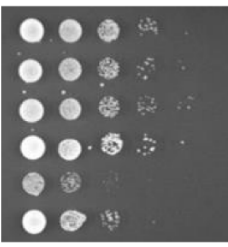

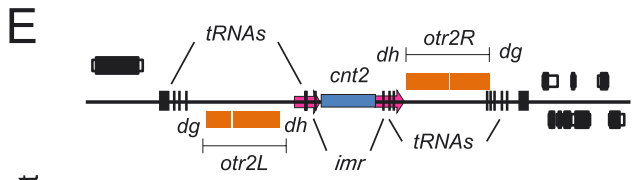

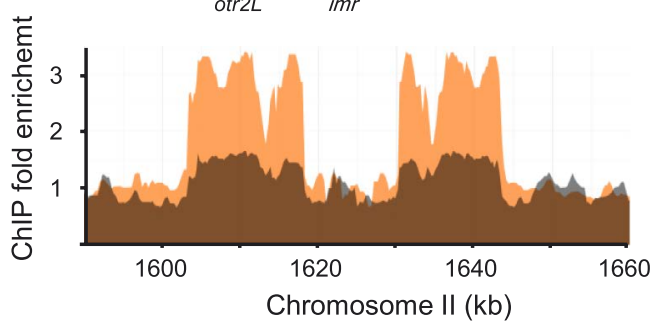

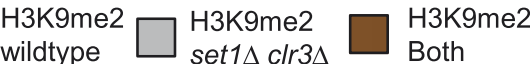

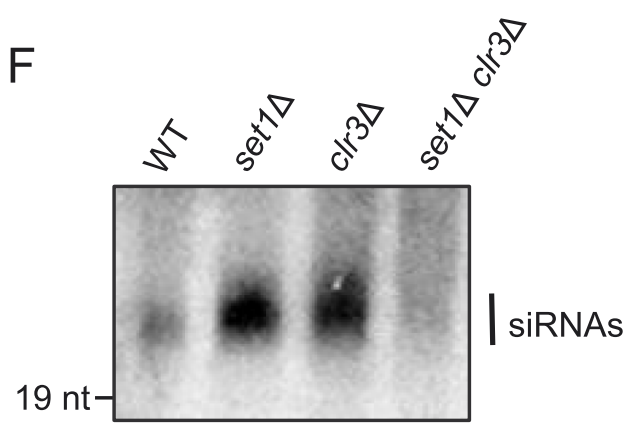

Figure 4. Set1 and the class II HDAC Clr3 cooperates in heterochromatic silencing and heterochromatin formation. (A) Serial dilution analysis (SDA) of set1 and clr3 mutant strains in nonselective (N/S) media or in the presence of the tubulin inhibitor thiabendazole (TBZ), (B) uracil minus media (-Ura) or in the presence of the uracil counter selective drug 5-fluoroorotic acid (5-FOA). (C) Transcription of forward and reverse strands at centromere II in indicated mutant strains was analyzed by microarrays. (D) H3K9 dimethylation (H3K9me2) in strains deficient for set1 and clr3 at the pericentromeric dg repeat. $\mathrm{H} 3 \mathrm{~K} 9 \mathrm{me} 2$ enrichment at the dg repeat in indicated strains was carried out by chromatin immunoprecipitation (ChIP) and quantified by qPCR. (E) H3K9me2 Figure 4. Continued on next page 
Figure 4. Continued

distribution across the entire centromere II in wild-type and set1 $\Delta$ clr3 $\Delta$ strains. H3K9me2 at centromere II was assayed by ChIP-chip. (F) siRNA levels in wild-type, set1 and clr3 mutant strains. Detection of siRNAs was carried out by a northern blot using a probe specific for pericentromeric dg repeats. DOI: 10.7554/eLife.04506.015

The following figure supplements are available for figure 4:

Figure supplement 1. Pol II and Swib localization at pericentromeres in set1 and clr3 mutants.

DOI: 10.7554/eLife.04506.016

Figure supplement 2. H3K9me2 defects at centromeres I and III, mating type locus and subtelomeric regions in a strain deficient for both set1 and clr3. DOI: 10.7554/eLife.04506.017

inhibitor thiabendazole (Figure 4A), suggesting defects in chromosome segregation. Importantly, the set1 $F^{H 3 K 4 m e-~} c l r 3 \Delta$ double mutant, in which set1 has no H3K4me activity, exhibited only slight defects. Derepression of a reporter gene inserted within the pericentromeric repeats has been observed in mutants deficient for either set1 (Kanoh et al., 2003) or clr3 (Grewal et al., 1998). We observed additional derepression of the reporter gene in mutants deficient for both set1 and clr3 (Figure 4B). Defects in heterochromatic silencing result in transcriptional derepression of both the forward and reverse strands of pericentromeric repeats (Volpe et al., 2002; Moazed, 2011; Alper et al., 2013). We performed expression analysis using tiling microarrays to assess transcription on both strands in set1 and clr3 mutant strains. Modest increases in transcript levels were found on both strands associated with the pericentromeric $d g$ and $d h$ repeats in single set $1 \Delta$ and clr3 $\Delta$ mutants. However, in the set $1 \Delta$ clr3 $\Delta$ double mutant, the increase was not only synergistic but occurred throughout the entire pericentromeric region (Figure $4 C$ ).

Heterochromatin assembly is characterized by the establishment of histone $\mathrm{H} 3$ lysine 9 methylation (H3K9me) and HP1/Swib proteins bound to H3K9me (Nakayama et al., 2001). H3K9me/Swi6 is thought to provide a platform for the recruitment of histone modifiers such as HDACs which could restrict the accessibility of Pol II (Yamada et al., 2005). We performed chromatin immunoprecipitation (ChIP) followed by quantitative PCR (qPCR) to monitor the levels of H3K9me, Swi6, and Pol II at the pericentromeric $d g$ repeats in the set 1 and clr3 mutants. Similar to previous observations (Yamada et al., 2005), the loss of clr3 resulted in increased levels of H3K9me2 and Pol II and a decrease in Swi6 enrichment (Figure 4D; Figure 4-figure supplement 1). Loss of set1 resulted in a slight increase of Pol II localization (Xhemalce and Kouzarides, 2010) and did not diminish H3K9me2 and Swi6 levels at the dg repeats. In contrast, there was a dramatic reduction in the levels of H3K9me2 and Swi6 accompanied by further increase of Pol II occupancy in the double mutant lacking both set1 and clr3. We extended our analysis of H3K9me2 genome-wide and found that H3K9me2 levels in set1 $\Delta$ clr3 $\Delta$ mutant were reduced across the entire pericentromeric region (Figure 4E). H3K9me2 defects in the double mutant were seen at other centromeres and heterochromatin domains, including the silent mating type region and subtelomeres (Figure 4-figure supplement 2). The RNAi machinery is known to contribute to the assembly of pericentromeric heterochromatin, in part by acting in cis to generate siRNAs (Volpe et al., 2002; Noma et al., 2004). We found that whereas loss of clr3 or set 1 resulted in an increase of siRNAs (Sugiyama et al., 2007), the level of siRNAs was dramatically reduced in the double mutant (Figure 4F). Thus, our results reveal compensatory mechanisms by Set1 and Clr3 acting in parallel pathways to maintain heterochromatin at major chromosomal landmarks in S. pombe.

\section{Coordinated repression by Set1 and Clr3 on a substantial portion of the S. pombe transcriptome}

To assess the extent of functional cooperation between Set1 and Clr3 in controlling transcription genome-wide, we performed comparative transcriptome analysis in set 1 and clr3 mutant cells. While the majority of the differentially expressed probes in the set $1 \Delta$ mutant corresponded to increased expression, loss of clr3 resulted in 792 probes changing significantly in comparison with wild-type, with approximately equal numbers corresponding to upregulated and downregulated transcripts (Figure 5A). Intriguingly, cells lacking both set 1 and clr3 displayed differential expression of nearly 2900 probes, 2343 of which were upregulated. Loss of H3K4me in a clr3 null background (set1 $F^{H 3 K 4 m e-}$ clr3 $\Delta$ ) did not produce such a drastic change to the transcriptome compared with set $1 \Delta \operatorname{clr} 3 \Delta$, but only reduced the proportion of downregulated transcripts seen in the single clr3 $\Delta$ mutant. Similar proportions of probes corresponding to the sense or antisense strands of known transcripts were differentially expressed across set $1 \Delta$ and set $1 \Delta \operatorname{clr} 3 \Delta$ mutants, with the exception of $c / r 3 \Delta$ cells, which displayed an increased 


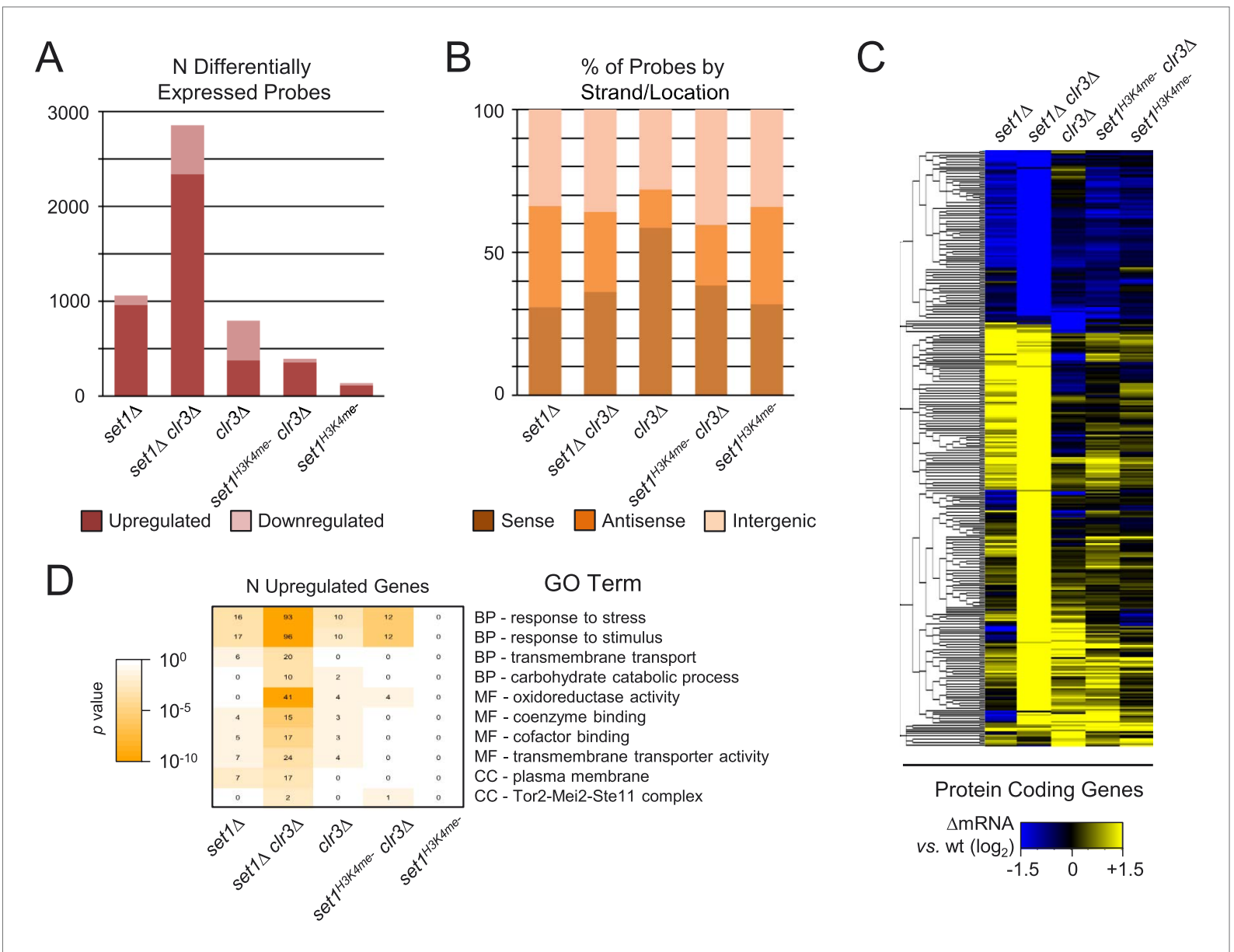

Figure 5. Upregulation of a large fraction of the transcriptome in a strain deficient for both set1 and clr3. (A) Counts and (B) percentage of probes matching feature strand/position in indicated mutant strains were analyzed similarly to Figure $\mathbf{2 A}$ and $\mathbf{B}$. (C) Hierarchical clustering of significantly changed protein coding genes in set 1 and clr3 mutant gene expression profiles $(n=346)$. Sense strand probes from two microarray experiments were averaged and clustered as in Figure 2C. (D) Gene ontology (GO) analysis of upregulated transcripts in set1 and clr3 mutant gene expression microarrays. Representative GO terms from biological process ('BP'), molecular function ('MF'), and cellular component ('CC') ontologies displaying most significant enrichment (right panel) and corresponding number of upregulated genes (left panel) in indicated mutant strains; all enriched terms are listed in Figure 5-source data 1 p values, hypergeometric test.

DOI: 10.7554/eLife.04506.018

The following source data and figure supplements are available for figure 5:

Source data 1. Gene ontology (GO) term enrichment in set1/clr3 mutant expression profiling microarrays.

DOI: 10.7554/eLife.04506.019

Figure supplement 1. Representative genes whose expression requires set1 and clr3.

DOI: 10.7554/eLife.04506.020

Figure supplement 2. Synergistic upregulation of Tf2s and subtelomeric regions in strain deficient for both set1 and clr3.

DOI: 10.7554/eLife.04506.021

Figure supplement 3. Set1 and $\mathrm{Clr} 3$ cooperate to control genes involved in the core environmental stress response.

DOI: 10.7554/eLife.04506.022

Figure supplement 4. Cooperation between Set1 and Clr3 in development.

DOI: 10.7554/eLife.04506.023

proportion of sense strand probes (Figure 5B). Hierarchical clustering showed that transcripts downregulated in set1 $\Delta$ tended to be downregulated further in set1 $\Delta$ clr3 $\Delta$ (Figure 5-figure supplement 1), and transcripts that were upregulated in set $1 \Delta$ (i.e., Tf2s and subtelomeric regions) were further upregulated in the double mutants (Figure 5C; Figure 5-figure supplement 2). Most notably, loss of both set 1 and $c / r 3$ resulted in significant expression changes within protein-coding gene regions for a large subset of genes displaying negligible change in individual set1 or clr3 mutants (Figure 5C). 
Upregulated transcripts include well-characterized developmental and stress-response regulatory proteins that include fbp1, mei2 and ste11 (Figure 5-figure supplement 3). Gene ontology analysis suggested that most of the upregulated transcripts in set $1 \Delta \mathrm{clr} 3 \Delta$ are associated with stress-response processes that include the Tor2-Mei2-Ste11 pathways (Figure 5D; Figure 5-source data 1). These pathways are known to be activated during the meiotic development program (Otsubo and Yamamoto, 2012). In this regard, we noted that compared with wild-type or single mutant strains, the set1 $\Delta$ clr3 $\Delta$ double mutant exhibited considerable meiotic defects (Figure 5-figure supplement 4). Collectively, our results disclose unexpected coordination between Set 1 and $\mathrm{Clr} 3$ in ensuring genome-wide repression of the fission yeast transcriptome and proper developmental control.

\section{Discussion}

\section{Set1C as a repressor complex of the fission yeast transcriptome}

Recent transcriptome studies of chromatin mutants in $S$. cerevisiae reveal that loss of set 1 or any of the other four core Set1C subunits (Swd1, Swd3, Bre2/Ash2, Sdc1) produces comparable expression profiles (Margaritis et al., 2012). Furthermore, loss of set1 has only a modest effect on the transcriptome, mainly towards derepression that could fully be accounted by the loss of H3K4me (Margaritis et al., 2012; Weiner et al., 2012). Similar to these studies, our current study shows that complete loss of $\mathrm{H} 3 \mathrm{~K} 4 \mathrm{me}$ (i.e., H3K4R, set $1 \mathrm{~F}^{\mathrm{H} K \mathrm{~K} 4 \mathrm{me}-}$ mutants) in S. pombe has only a slight impact on the transcriptome, with most differentially expressed transcripts upregulated. However, there are important differences. Except for the expression profiles of $H 3 K 4 R$ and set1 $F^{H 3 K 4 m e-}$ mutants, the profiles among $S$. pombe Set1C subunit mutants are notably disparate, which could not be fully explained by their roles as subunits of Set1C or contributions to H3K4me (Roguev et alo, 2003). For example, Ash2 and Sdc1 are thought to form heterodimers that together with Swd1 and Swd3 constitute the core of the Set1C complex (Roguev et al., 2001; Dehe et al., 2006; Southall et al., 2009; Kim et al., 2013). Yet, while their expression profiles are most similar to each other, there are even differences between them, with the sdc1 mutant displaying stronger derepression for a subset of genes involved in response to oxidative stress than those seen in the ash2 mutant (Figure 1C). These similarities and differences might reflect their association with other chromatin modifiers such as the Lid2 complex, not present in budding yeast (Roguev et al., 2003; Shevchenko et al., 2008). Most importantly, the expression profile of set1 $\Delta$ is strikingly different from those of other Set1C/H3K4me mutants, displaying more than eight times the number of upregulated probes relative to those of swd3 or H3K4R mutants. Our findings show that unlike the results reported for $S$. cerevisiae, Set1 in $S$. pombe not only exerts more regulatory influence over the transcriptome, but also mediates its repressive function largely independently of the other Set1C subunits and H3K4 methylation-probably, as a consequence of the uncoupling of Set1 protein stability from H3K4me levels (Mikheyeva et al., 2014). Interestingly, S. pombe Set1 has been reported as a component of at least two complexes: a large $\sim 1 \mathrm{MDa}$ complex similar in size to that of S. cerevisiae Set1C and a smaller complex $(\sim 800 \mathrm{kDa})$ containing a shorter version of Set1 (Roguev et al., 2003). Thus, Set1 might mediate its repressive nonH3K4me function via a distinct form of Set1 different from the form associated with the canonical Set1C complex.

\section{Regulation of repetitive elements, developmental and stress-response loci by Set1 and Atf1}

Our study reveals extensive functional interactions across the genome between Set 1 and the stressresponse transcription factor Atf1 at stress-response genes and major chromosomal landmarks, including the tandem rDNA array and centromeres. At the rDNA array and centromere central cores, Atf1 mediates Set1 recruitment and modulates H3K4me3 levels that might contribute to proper chromatin organization rather than transcriptional repression itself. At loci of stress response and developmental regulators such as ste11, Atf1 and Set1 appear to act in parallel pathways that contribute to the repression of ste11 as loss of both atf1 and set 1 resulted in significant derepression of ste11 (Figure 3-figure supplement 4). The transcriptional activation of Atf1 is controlled by phosphorylation mediated by the stress-activated mitogen-activated protein kinase (MAPK) Sty1 pathway (Shiozaki and Russell, 1996; Lawrence et al., 2007). It is likely that co-occupancy of Set1 and Atf1 at the promoters of certain developmental and stress-response regulators not only helps keep these genes in a poised transcriptional off-state, but might also contribute to their rapid transcriptional activation in response to proper developmental or environmental stress signals. 


\section{Functional cooperation between Set1 and Clr3 in heterochromatic silencing and genome-wide repression of the transcriptome}

Pol II activity is known to be required for transcriptional silencing and heterochromatin assembly at pericentromeric repeats (Djupedal et al., 2005; Kato et al., 2005). Other factors associated with active Pol II transcription including components of the Mediator complex have also been shown to contribute to heterochromatin formation (Oya et al., 2013). Our study identifies an important role for Set1 in the assembly of heterochromatin domains such as those present at pericentromeres (Figure 6). Set1 represses transcription on both the forward and reverse strands of the pericentromeric repeats and cooperates with $\mathrm{Clr} 3$ to assemble $\mathrm{H} 3 \mathrm{~K} 9$ me-associated hetero-

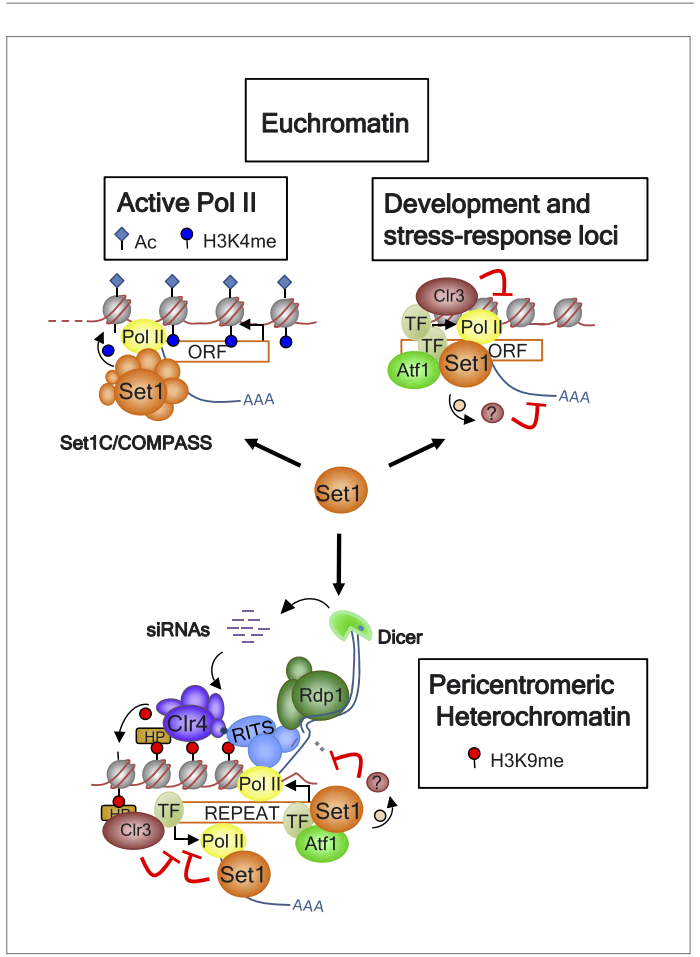

Figure 6. Model for Set1 functions at euchromatin and heterochromatin domains. At euchromatin domains, the Set1C/COMPASS complex is recruited to active Pol II genes and provides the H3K4me marks. Set1 is also recruited to certain lowly expressed and repressed genes associated with developmental and stressresponse pathways in part by Atf1, other transcription factors (TFs), and probably transcriptionally poised Pol II. Set1 acts in a parallel pathway with the histone deacetylase (HDAC) Clr3 to impose transcriptional repression at these loci. At a heterochromatin domain such as the pericentromeric region, Atf1 and probably other unidentified TFs mediate the recruitment of Set1 to sites enriched for tRNAs known to act as boundary elements. Set 1 coordinates with $\mathrm{Clr} 3$ in the establishment of SUV39H1/Clr4-mediated H3K9me/HP1 (HP: Swib and Chp2) heterochromatin and suppression of bidirectional transcription independently of $\mathrm{H} 3 \mathrm{~K} 4 \mathrm{me}$ and the other Set1C subunits. Set1-mediated silencing could occur via methylation of nonhistone substrate(s) through the same or different pathways from those of RNAi (i.e., RITS, Rdp1, Dicer) or the exosome (not shown)

DOI: 10.7554/eLife.04506.024 chromatin. Importantly, this heterochromatic activity of Set1 appears to be independent of its canonical H3K4me function associated with the Set1C complex, consistent with previous observations for the general lack of H3K4me within H3K9me heterochromatin (Noma et al., 2001; Cam et al., 2005). Set1-mediated heterochromatin assembly might involve Set1 methylating a nonhistone substrate similar to that of SUV39H1/Clr4 methylating Mlo3, an RNA processing and nuclear export factor that also contributes to RNAi-mediated heterochromatin assembly (Zhang et al., 2011). The only known nonhistone target of Set1 is the kinetochore protein DAM1 in S. cerevisiae (Zhang et al., 2005). However, the $S$. pombe dam 1 ortholog does not appear to be the target of Set1-mediated heterochromatic silencing as repression of Tf2 retrotransposons and pericentromeric heterochromatin is maintained in dam 1 mutant cells (Mikheyeva and Cam, unpublished data).

In addition to heterochromatic repeats, a significant fraction of the transcriptome is under repressive control by Set 1 and $\mathrm{Clr} 3$. Such genome-wide repressive effect strongly suggests that Set1 behaves largely as a bona fide repressor. At developmental and stress-response loci such as ste11, Set1 may act in concert with transcription factors, including Atf1 together with $\mathrm{Clr} 3$ and other HDACs, to keep the target genes repressed in a steady-state condition. However, unlike heterochromatin, the chromatin states of these loci probably support a transcriptionally poised Pol II and in response to appropriate environmental signals enable Pol II to rapidly upregulate transcription.

\section{Materials and methods}

\section{Strain Construction}

Null mutants of Set1C subunits were constructed using a kanamycin cassette (Bahler et al., 1998; Mikheyeva et al., 2014). Double mutants were generated by standard genetic cross methods (Moreno et alo, 1991). Liquid cultures were grown at $30^{\circ} \mathrm{C}$ in standard rich media supplemented with $75 \mathrm{mg} / \mathrm{l}$ adenine (YEA). 


\section{Chromatin immunoprecipitation (ChIP) and ChIP-chip}

ChIP assays were performed as previously described (Lorenz et al., 2012). ChIP enrichment was quantified by qPCR analysis. ChIP-chip was carried out as previously described using Agilent tiling microarrays (Cam et al., 2005). ChIP-chip analysis was performed using the R/Bioconductor ringo package (Toedling et al., 2007). Preprocessing was carried out by loess normalization. ChIP-enriched regions were defined as three or more adjacent microarray probes with fold-enrichment greater than a two-Gaussian null distribution threshold (greater than twofold enrichment). Between-array analysis of $\mathrm{H} 3 \mathrm{~K} 4 \mathrm{me} 3$ in wild-type and atf1 $\Delta$ experiments was performed using the limma (linear models for microarray data) package after interarray quantile normalization. Antibodies used for ChIP and ChIPchip assays were anti-FLAG Set1 (M2; Sigma-Aldrich, St. Louis, MO), anti-Atf1 (sc-53172; Santa Cruz Biotechnology, Inc., Dallas, Texas), anti Pol II (ab5408; Abcam, Cambridge, MA), anti-H3K4me3 (07-473; Millipore, Billerica, MA), anti-H3K9me2 (ab1220; Abcam), and anti-Swi6 (Nakayama et al., 2000).

\section{SiRNA detection}

Small RNAs were purified from $50 \mathrm{ml}$ culture of logarithmically growing cells using the Ambion mirVana miRNA/siRNA isolation kit (Life Technologies, Grand Island, NY). Small RNAs (60 $\mu$ g) were loaded onto a $15 \%$ denaturing polyacrylamide gel and run at $300 \mathrm{~V}$ until the bromophenol blue dye reached the bottom of the gel $(\sim 1.5 \mathrm{hr})$. Northern transfer was done overnight by capillary blotting in Trisborate-EDTA buffer at room temperature onto Hybond-N+ membrane (GE Healthcare, Pittsburgh, PA). The membrane was subsequently UV crosslinked twice at $1200 \mathrm{~J}$. Hybridization was carried out in $10 \mathrm{ml}$ ULTRAhyb-Oligo buffer (Life Technologies) at $40^{\circ} \mathrm{C}$ overnight with a ${ }^{32}$ P-labeled RNA probe specific to pericentromeric dg repeats. The RNA probe was generated by in vitro transcription using a T7 RNA polymerase system and $50 \mu \mathrm{Ci}$ of [ $\alpha{ }^{32}$ P]UTP. Detection of the siRNA signals was carried out using the Storm 820 molecular imager (Molecular Dynamics; GE Healthcare).

\section{Gene expression profiling}

Transcriptional profiling analysis was done as previously described (Lorenz et al., 2012). Briefly, RNA was extracted from batch cultures of mid-exponential phase $\left(\mathrm{OD}_{595} \sim 0.3-0.6\right)$ from mutant and isogenic wild-type strains, reverse-transcribed into cDNA, and labeled with either Alexa Fluor 555 (wild-type sample) or Alexa Fluor 647 (mutant sample) using Superscript Indirect cDNA labeling system (Life Technologies). Equal amounts of labeled cDNA (200-300 ng) from wild-type and mutant samples were mixed and hybridized on a custom $4 \times 44 \mathrm{k}$ probe Agilent tiling microarray as previously described (Cam et al., 2005). For hierarchical clustering using the R/Bioconductor hopach package (van der Laan and Pollard, 2003), interarray quantile normalization was performed using the limma package, and transcripts with more than one differentially expressed probe were averaged. The cosine angle function was used for the clustering distance metric. Gene Ontology (GO) enrichment was performed as previously described (Lorenz et al., 2012).

Datasets associated with transcriptional profiling and ChIP-chip experiments in this study can be accessed at the Gene Expression Omnibus under accession number GSE63301.

\section{Acknowledgements}

We thank Grace Kim, Daniel Shams, and Betty Slinger for experimental support, Ke Zhang for the siRNA protocol, Shiv Grewal for the Swi6 antibody, Ee Sin Chen, Irina Mikheyeva, and David Layman for critical reading of the manuscript. Work in the Cam laboratory is supported by the Boston College Wielers Faculty Research Fund and the March of Dimes Basil O' Connor Starter Scholar Research Award.

\section{Additional information}

Funding

\begin{tabular}{ll} 
Funder & Author \\
\hline March of Dimes Foundation & Hugh P Cam \\
\hline Boston College & Hugh P Cam \\
\hline
\end{tabular}

The funders had no role in study design, data collection and interpretation, or the decision to submit the work for publication. 
Author contributions

DRL, HPC, Conception and design, Acquisition of data, Analysis and interpretation of data, Drafting or revising the article; LFM, PJRG, Acquisition of data, Analysis and interpretation of data, Drafting or revising the article; MMM, Conception and design, Acquisition of data, Drafting or revising the article

\section{Additional files}

Major datasets

The following dataset was generated:

\begin{tabular}{|c|c|c|c|c|}
\hline Author(s) & Year & Dataset title & Dataset ID and/or URL & $\begin{array}{l}\text { Database, license, } \\
\text { and accessibility } \\
\text { information }\end{array}$ \\
\hline $\begin{array}{l}\text { Lorenz DR, Meyer LF, } \\
\text { Cam HP }\end{array}$ & 2014 & $\begin{array}{l}\text { Heterochromatin } \\
\text { assembly and } \\
\text { transcriptome } \\
\text { repression by Set1 } \\
\text { in coordination with } \\
\text { a class II histone } \\
\text { deacetylase }\end{array}$ & $\begin{array}{l}\text { http://www.ncbi.nlm.nih. } \\
\text { gov/geo/query/acc. } \\
\text { cgi?acc=GSE63301 }\end{array}$ & $\begin{array}{l}\text { Publicly available at } \\
\text { NCBI Gene Expression } \\
\text { Omnibus. }\end{array}$ \\
\hline
\end{tabular}

Reporting Standards: Standard used to collect data: Microarray datasets are deposited at NCBI GEO according to MIAME 2.0 standards.

The following previously published datasets were used:

\begin{tabular}{|c|c|c|c|c|}
\hline Author(s) & Year & Dataset title & Dataset ID and/or URL & $\begin{array}{l}\text { Database, license, } \\
\text { and accessibility } \\
\text { information }\end{array}$ \\
\hline $\begin{array}{l}\text { Zaratiegui M, Vaughn MW, } \\
\text { Irvine DV, Goto D, Watt S, } \\
\text { Bahler J, Arcangioli B, } \\
\text { Martienssen RA }\end{array}$ & 2010 & $\begin{array}{l}\text { CENP-B preserves } \\
\text { genome integrity at } \\
\text { replication forks paused } \\
\text { by retrotransposon LTR }\end{array}$ & $\begin{array}{l}\text { http://www.ncbi.nlm.nih. } \\
\text { gov/sra/?term=SRA024710.2 }\end{array}$ & $\begin{array}{l}\text { Publicly available at } \\
\text { NCBI Sequence Read } \\
\text { Archive. }\end{array}$ \\
\hline $\begin{array}{l}\text { Rhind N, Chen Z, } \\
\text { Yassour M, Thompson DA, } \\
\text { Haas BJ, Habib N, }\end{array}$ & 2011 & $\begin{array}{l}\text { Comparative functional } \\
\text { genomics of the fission } \\
\text { yeasts }\end{array}$ & $\begin{array}{l}\text { http://www.ncbi.nlm.nih. } \\
\text { gov/sra/?term=SRP005611 }\end{array}$ & $\begin{array}{l}\text { Publicly available at } \\
\text { NCBI Sequence Read } \\
\text { Archive. }\end{array}$ \\
\hline
\end{tabular}

Wapinski I, Roy S,

Lin MF, Heiman DI

\section{References}

Alper BJ, Job G, Yadav RK, Shanker S, Lowe BR, Partridge JF. 2013. Sir2 is required for Clr4 to initiate centromeric heterochromatin assembly in fission yeast. The EMBO Journal 32:2321-2335. doi: 10.1038/ emboj.2013.143.

Badeaux Al, Shi Y. 2013. Emerging roles for chromatin as a signal integration and storage platform. Nature Reviews Molecular Cell Biology 14:211-224. doi: 10.1038/nrm3545.

Bähler J, Wu JQ, Longtine MS, Shah NG, McKenzie A III, Steever AB, Wach A, Philippsen P, Pringle JR. 1998. Heterologous modules for efficient and versatile PCR-based gene targeting in Schizosaccharomyces pombe. Yeast 14:943-951. doi: 10.1002/(SICI)1097-0061(199807)14:10<943::AID-YEA292>3.0.CO;2-Y.

Berretta J, Pinskaya M, Morillon A. 2008. A cryptic unstable transcript mediates transcriptional transsilencing of the Ty1 retrotransposon in S. cerevisiae. Genes \& Development 22:615-626. doi: 10.1101/ gad.458008.

Bühler M, Haas W, Gygi SP, Moazed D. 2007. RNAi-dependent and -independent RNA turnover mechanisms contribute to heterochromatic gene silencing. Cell 129:707-721. doi: 10.1016/j.cell.2007.03.038.

Cam HP, Noma K, Ebina H, Levin HL, Grewal SI. 2008. Host genome surveillance for retrotransposons by transposon-derived proteins. Nature 451:431-436. doi: 10.1038/nature06499.

Cam HP, Sugiyama T, Chen ES, Chen X, FitzGerald PC, Grewal SI. 2005. Comprehensive analysis of heterochromatin- and RNAi-mediated epigenetic control of the fission yeast genome. Nature Genetics 37:809-819. doi: 10.1038/ng1602.

Camblong J, Beyrouthy N, Guffanti E, Schlaepfer G, Steinmetz LM, Stutz F. 2009. Trans-acting antisense RNAs mediate transcriptional gene cosuppression in S. cerevisiae. Genes \& Development 23:1534-1545. doi: 10.1101/gad.522509.

Chen D, Toone WM, Mata J, Lyne R, Burns G, Kivinen K, Brazma A, Jones N, Bähler J. 2003. Global transcriptional responses of fission yeast to environmental stress. Molecular Biology of the Cell 14:214-229. doi: 10.1091/mbc E02-08-0499. 
Chen ES, Zhang K, Nicolas E, Cam HP, Zofall M, Grewal SI. 2008. Cell cycle control of centromeric repeat transcription and heterochromatin assembly. Nature 451:734-737. doi: 10.1038/nature06561.

Dehé PM, Dichtl B, Schaft D, Roguev A, Pamblanco M, Lebrun R, Rodríguez-Gil A, Mkandawire M, Landsberg K, Shevchenko A, Shevchenko A, Rosaleny LE, Tordera V, Chávez S, Stewart AF, Géli V. 2006. Protein interactions within the Set1 complex and their roles in the regulation of histone 3 lysine 4 methylation. The Journal of Biological Chemistry 281:35404-35412. doi: 10.1074/jbc.M603099200.

Djupedal I, Portoso M, Spåhr H, Bonilla C, Gustafsson CM, Allshire RC, Ekwall K. 2005. RNA Pol Il subunit Rpb7 promotes centromeric transcription and RNAi-directed chromatin silencing. Genes \& Development 19:2301-2306. doi: 10.1101/gad.344205.

Eshaghi M, Lee JH, Zhu L, Poon SY, Li J, Cho KH, Chu Z, Karuturi RK, Liu J. 2010. Genomic binding profiling of the fission yeast stress-activated MAPK Sty1 and the bZIP transcriptional activator Atf1 in response to $\mathrm{H} 2 \mathrm{O} 2$. PLOS ONE 5:e11620. doi: 10.1371/journal.pone.0011620.

Grewal SI, Bonaduce MJ, Klar AJ. 1998. Histone deacetylase homologs regulate epigenetic inheritance of transcriptional silencing and chromosome segregation in fission yeast. Genetics 150:563-576.

Grewal SI, Jia S. 2007. Heterochromatin revisited. Nature Reviews Genetics 8:35-46. doi: 10.1038/nrg2008.

Grunstein M. 1998. Yeast heterochromatin: regulation of its assembly and inheritance by histones. Cell 93:325-328. doi: 10.1016/S0092-8674(00)81160-5.

Gullerova M, Proudfoot NJ. 2008. Cohesin complex promotes transcriptional termination between convergent genes in S. pombe. Cell 132:983-995. doi: 10.1016/j.cell.2008.02.040.

Hansen KR, Burns G, Mata J, Volpe TA, Martienssen RA, Bähler J, Thon G. 2005. Global effects on gene expression in fission yeast by silencing and RNA interference machineries. Molecular and Cellular Biology 25:590-601. doi: 10.1128/MCB.25.2.590-601.2005.

Heintzman ND, Stuart RK, Hon G, Fu Y, Ching CW, Hawkins RD, Barrera LO, Van Calcar S, Qu C, Ching KA, Wang W, Weng Z, Green RD, Crawford GE, Ren B. 2007. Distinct and predictive chromatin signatures of transcriptional promoters and enhancers in the human genome. Nature Genetics 39:311-318. doi: 10.1038/ ng1966.

Henikoff S, Shilatifard A. 2011. Histone modification: cause or cog? Trends in Genetics 27:389-396. doi: 10.1016/ j.tig.2011.06.006.

Huisinga KL, Brower-Toland B, Elgin SC. 2006. The contradictory definitions of heterochromatin: transcription and silencing. Chromosoma 115:110-122. doi: 10.1007/s00412-006-0052-x.

Jia S, Noma K, Grewal SI. 2004. RNAi-independent heterochromatin nucleation by the stress-activated ATF/CREB family proteins. Science 304:1971-1976. doi: 10.1126/science.1099035.

Kanoh J, Francesconi S, Collura A, Schramke V, Ishikawa F, Baldacci G, Géli V. 2003. The fission yeast spSet1p is a histone $\mathrm{H} 3-\mathrm{K} 4$ methyltransferase that functions in telomere maintenance and DNA repair in an ATM kinase Rad3-dependent pathway. Journal of Molecular Biology 326:1081-1094. doi: 10.1016/S0022-2836(03)00030-5.

Kato H, Goto DB, Martienssen RA, Urano T, Furukawa K, Murakami Y. 2005. RNA polymerase II is required for RNAi-dependent heterochromatin assembly. Science 309:467-469. doi: 10.1126/science.1114955.

Kim T, Buratowski S. 2009. Dimethylation of H3K4 by Set1 recruits the Set3 histone deacetylase complex to 5 transcribed regions. Cell 137:259-272. doi: 10.1016/j.cell.2009.02.045.

Kim J, Kim JA, McGinty RK, Nguyen UT, Muir TW, Allis CD, Roeder RG. 2013. The n-SET domain of Set1 regulates H2B ubiquitylation-dependent H3K4 methylation. Molecular Cell 49:1121-1133. doi: 10.1016/j.molcel.2013.01.034.

Kouzarides T. 2007. Chromatin modifications and their function. Cell 128:693-705. doi: 10.1016/j.cell. 2007.02.005.

Krogan NJ, Dover J, Khorrami S, Greenblatt JF, Schneider J, Johnston M, Shilatifard A. 2002. COMPASS, a histone H3 (Lysine 4) methyltransferase required for telomeric silencing of gene expression. The Journal of Biological Chemistry 277:10753-10755. doi: 10.1074/jbc.C200023200.

Krogan NJ, Dover J, Wood A, Schneider J, Heidt J, Boateng MA, Dean K, Ryan OW, Golshani A, Johnston M, Greenblatt JF, Shilatifard A. 2003. The Paf1 complex is required for histone H3 methylation by COMPASS and Dot1p: linking transcriptional elongation to histone methylation. Molecular Cell 11:721-729. doi: 10.1016/ S1097-2765(03)00091-1.

Kusch T. 2012. Histone H3 lysine 4 methylation revisited. Transcription 3:310-314. doi: 10.4161/trns.21911.

Lawrence CL, Maekawa H, Worthington JL, Reiter W, Wilkinson CR, Jones N. 2007. Regulation of Schizosaccharomyces pombe Atf1 protein levels by Sty1-mediated phosphorylation and heterodimerization with Pcr1. The Journal of Biological Chemistry 282:5160-5170. doi: 10.1074/jbc.M608526200.

Litt MD, Simpson M, Gaszner M, Allis CD, Felsenfeld G. 2001. Correlation between histone lysine methylation and developmental changes at the chicken beta-globin locus. Science 293:2453-2455. doi: 10.1126/ science.1064413.

Lorenz DR, Mikheyeva IV, Johansen P, Meyer L, Berg A, Grewal SI, Cam HP. 2012. CENP-b cooperates with Set1 in bidirectional transcriptional silencing and genome organization of retrotransposons. Molecular and Cellular Biology 32:4215-4225. doi: 10.1128/MCB.00395-12.

Margaritis T, Oreal V, Brabers N, Maestroni L, Vitaliano-Prunier A, Benschop JJ, van Hooff S, van Leenen D, Dargemont C, Géli V, Holstege FC. 2012. Two Distinct Repressive Mechanisms for Histone 3 Lysine 4 Methylation through Promoting 3'-End Antisense Transcription. PLOS Genetics 8:e1002952. doi: 10.1371/ journal.pgen.1002952.

Mikheyeva IV, Grady PJ, Tamburini FB, Lorenz DR, Cam HP. 2014. Multifaceted genome control by Set1 dependent and independent of H3K4 methylation and the Set1C/COMPASS complex. PLOS Genetics 10:e1004740. doi: 10.1371/journal.pgen.1004740. 
Moazed D. 2011. Mechanisms for the inheritance of chromatin states. Cell 146:510-518. doi: 10.1016/j.cell. 2011.07.013.

Moreno S, Klar A, Nurse P. 1991. Molecular genetic analysis of fission yeast Schizosaccharomyces pombe. Methods in Enzymology 194:795-823. doi: 10.1016/0076-6879(91)94059-L.

Nakayama J, Klar AJ, Grewal SI. 2000. A chromodomain protein, Swi6, performs imprinting functions in fission yeast during mitosis and meiosis. Cell 101:307-317. doi: 10.1016/S0092-8674(00)80840-5.

Nakayama J, Rice JC, Strahl BD, Allis CD, Grewal SI. 2001. Role of histone H3 lysine 9 methylation in epigenetic control of heterochromatin assembly. Science 292:110-113. doi: 10.1126/science.1060118.

$\mathrm{Ng} \mathrm{HH}$, Robert F, Young RA, Struhl K. 2003. Targeted recruitment of Set1 histone methylase by elongating Pol II provides a localized mark and memory of recent transcriptional activity. Molecular Cell 11:709-719. doi: 10.1016/S1097-2765(03)00092-3.

Nislow C, Ray E, Pillus L. 1997. SET1, a yeast member of the trithorax family, functions in transcriptional silencing and diverse cellular processes. Molecular Biology of the Cell 8:2421-2436. doi: 10.1091/mbc.8.12.2421.

Noma K, Allis CD, Grewal SI. 2001. Transitions in distinct histone H3 methylation patterns at the heterochromatin domain boundaries. Science 293:1150-1155. doi: 10.1126/science.1064150.

Noma K, Sugiyama T, Cam H, Verdel A, Zofall M, Jia S, Moazed D, Grewal SI. 2004. RITS acts in cis to promote RNA interference-mediated transcriptional and post-transcriptional silencing. Nature Genetics 36:1174-1180. doi: $10.1038 /$ ng1452.

Otsubo Y, Yamamoto M. 2012. Signaling pathways for fission yeast sexual differentiation at a glance. Journal of Cell Science 125:2789-2793. doi: 10.1242/jcs.094771.

Oya E, Kato H, Chikashige Y, Tsutsumi C, Hiraoka Y, Murakami Y. 2013. Mediator directs co-transcriptional heterochromatin assembly by RNA interference-dependent and -independent pathways. PLOS Genetics 9:e1003677. doi: 10.1371/journal.pgen.1003677.

Pokholok DK, Harbison CT, Levine S, Cole M, Hannett NM, Lee TI, Bell GW, Walker K, Rolfe PA, Herbolsheimer E, Zeitlinger J, Lewitter F, Gifford DK, Young RA. 2005. Genome-wide map of nucleosome acetylation and methylation in yeast. Cell 122:517-527. doi: 10.1016/j.cell.2005.06.026.

Rando OJ, Chang HY. 2009. Genome-wide views of chromatin structure. Annual Review of Biochemistry 78:245-271. doi: 10.1146/annurev.biochem.78.071107.134639.

Reyes-Turcu FE, Zhang K, Zofall M, Chen E, Grewal SI. 2011. Defects in RNA quality control factors reveal RNAi-independent nucleation of heterochromatin. Nature Structural \& Molecular Biology 18:1132-1138. doi: $10.1038 /$ nsmb.2122.

Rhind N, Chen Z, Yassour M, Thompson DA, Haas BJ, Habib N, Wapinski I, Roy S, Lin MF, Heiman DI, Young SK, Furuya K, Guo Y, Pidoux A, Chen HM, Robbertse B, Goldberg JM, Aoki K, Bayne EH, Berlin AM, Desjardins CA, Dobbs E, Dukaj L, Fan L, FitzGerald MG, French C, Gujja S, Hansen K, Keifenheim D, Levin JZ, Mosher RA, Müller CA, Pfiffner J, Priest M, Russ C, Smialowska A, Swoboda P, Sykes SM, Vaughn M, Vengrova S, Yoder R, Zeng Q, Allshire R, Baulcombe D, Birren BW, Brown W, Ekwall K, Kellis M, Leatherwood J, Levin H, Margalit $H$, Martienssen R, Nieduszynski CA, Spatafora JW, Friedman N, Dalgaard JZ, Baumann P, Niki H, Regev A, Nusbaum C. 2011. Comparative functional genomics of the fission yeasts. Science 332:930-936. doi: 10.1126/ science. 1203357.

Roguev A, Schaft D, Shevchenko A, Aasland R, Stewart AF 2003. High conservation of the Set1/Rad6 axis of histone 3 lysine 4 methylation in budding and fission yeasts. The Journal of Biological Chemistry 278:8487-8493. doi: 10.1074/jbc.M209562200.

Roguev A, Schaft D, Shevchenko A, Pijnappel WW, Wilm M, Aasland R, Stewart AF. 2001. The Saccharomyces cerevisiae Set1 complex includes an Ash2 homologue and methylates histone 3 lysine 4. The EMBO Journal 20:7137-7148. doi: 10.1093/emboj/20.24.7137.

Shevchenko A, Roguev A, Schaft D, Buchanan L, Habermann B, Sakalar C, Thomas H, Krogan NJ, Shevchenko A, Stewart AF. 2008. Chromatin Central: towards the comparative proteome by accurate mapping of the yeast proteomic environment. Genome Biology 9:R167. doi: 10.1186/gb-2008-9-11-r167.

Shilatifard A. 2012. The COMPASS family of histone H3K4 methylases: mechanisms of regulation in development and disease pathogenesis. Annual Review of Biochemistry 81:65-95. doi: 10.1146/annurev-biochem-051710134100.

Shiozaki K, Russell P. 1996. Conjugation, meiosis, and the osmotic stress response are regulated by Spc1 kinase through Atf1 transcription factor in fission yeast. Genes \& Development 10:2276-2288. doi: 10.1101/ gad.10.18.2276.

Southall SM, Wong PS, Odho Z, Roe SM, Wilson JR. 2009. Structural basis for the requirement of additional factors for MLL1 SET domain activity and recognition of epigenetic marks. Molecular Cell 33:181-191. doi: 10.1016/j.molcel.2008.12.029.

Sugiyama T, Cam HP, Sugiyama R, Noma K, Zofall M, Kobayashi R, Grewal S. 2007. SHREC, an effector complex for heterochromatic transcriptional silencing. Cell 128:491-504. doi: 10.1016/j.cell.2006.12.035.

Tashiro S, Asano T, Kanoh J, Ishikawa F. 2013. Transcription-induced chromatin association of RNA surveillance factors mediates facultative heterochromatin formation in fission yeast. Genes to Cells 18:327-339. doi: 10.1111/gtc.12038.

Toedling J, Skylar O, Krueger T, Fischer JJ, Sperling S, Huber W. 2007. Ringo-an R/Bioconductor package for analyzing ChIP-chip readouts. BMC Bioinformatics 8:221. doi: 10.1186/1471-2105-8-221.

van der Laan MJ, Pollard KS. 2003. A new algorithm for hybrid hierarchical clustering with visualization and the bootstrap. Journal of Statistical Planning and Inference 117:275-303. doi: 10.1016/S03783758(02)00388-9. 
van Dijk EL, Chen CL, d'Aubenton-Carafa Y, Gourvennec S, Kwapisz M, Roche V, Bertrand C, Silvain M, Legoix-Né P, Loeillet S, Nicolas A, Thermes C, Morillon A. 2011. XUTs are a class of Xrn1-sensitive antisense regulatory non-coding RNA in yeast. Nature 475:114-117. doi: 10.1038/nature10118.

Volpe TA, Kidner C, Hall IM, Teng G, Grewal SI, Martienssen RA. 2002. Regulation of heterochromatic silencing and histone H3 lysine-9 methylation by RNAi. Science 297:1833-1837. doi: 10.1126/science.1074973.

Weiner A, Chen HV, Liu CL, Rahat A, Klien A, Soares L, Gudipati M, Pfeffner J, Regev A, Buratowski S, Pleiss JA, Friedman N, Rando OJ. 2012. Systematic dissection of roles for chromatin regulators in a yeast stress response. PLOS Biology 10:e1001369. doi: 10.1371/journal.pbio.1001369.

Xhemalce B, Kouzarides T. 2010. A chromodomain switch mediated by histone H3 Lys 4 acetylation regulates heterochromatin assembly. Genes \& Development 24:647-652. doi: 10.1101/gad.1881710.

Yamada T, Fischle W, Sugiyama T, Allis CD, Grewal SI. 2005. The nucleation and maintenance of heterochromatin by a histone deacetylase in fission yeast. Molecular Cell 20:173-185. doi: 10.1016/j.molcel.2005.10.002.

Yamanaka S, Mehta S, Reyes-Turcu FE, Zhuang F, Fuchs RT, Rong Y, Robb GB, Grewal SI. 2013. RNAi triggered by specialized machinery silences developmental genes and retrotransposons. Nature 493:557-560. doi: 10.1038/ nature11716.

Yang XJ, Seto E. 2008. The Rpd3/Hda1 family of lysine deacetylases: from bacteria and yeast to mice and men. Nature Reviews Molecular Cell Biology 9:206-218. doi: 10.1038/nrm2346.

Zaratiegui M, Vaughn MW, Irvine DV, Goto D, Watt S, Bähler J, Arcangioli B, Martienssen RA. 2010. CENP-B preserves genome integrity at replication forks paused by retrotransposon LTR. Nature 469:112-115. doi: 10.1038/ nature09608.

Zhang K, Fischer T, Porter RL, Dhakshnamoorthy J, Zofall M, Zhou M, Veenstra T, Grewal SI. 2011. Clr4/Suv39 and RNA quality control factors cooperate to trigger RNAi and suppress antisense RNA. Science 331:1624-1627. doi: 10.1126/science.1198712.

Zhang K, Lin W, Latham JA, Riefler GM, Schumacher JM, Chan C, Tatchell K, Hawke DH, Kobayashi R, Dent SY. 2005. The Set1 methyltransferase opposes Ipl1 aurora kinase functions in chromosome segregation. Cell 122:723-734. doi: 10.1016/j.cell.2005.06.021.

Zofall M, Yamanaka S, Reyes-Turcu FE, Zhang K, Rubin C, Grewal SI. 2012. RNA elimination machinery targeting meiotic mRNAs promotes facultative heterochromatin formation. Science 335:96-100. doi: 10.1126/science. 1211651. 